\title{
Time-dependent quantum fluid density functional theory of hydrogen molecule under intense laser fields*
}

\author{
AMITA WADEHRA ${ }^{1}$ and $\mathrm{B} \mathrm{M} \mathrm{DEB}^{2, * *}$ \\ ${ }^{1}$ Department of Physics, Ohio State University, Columbus, OH 43210, USA \\ ${ }^{2}$ Indian Institute of Science Education and Research, HC-VII, Sector III, Salt Lake, Kolkata 700106 \\ e-mail: bmdeb@yahoo.co.in
}

MS received 29 May 2007; accepted 31 July 2007

\begin{abstract}
A time-dependent generalized non-linear Schrödinger equation (GNLSE) of motion was earlier derived in our laboratory by combining density functional theory and quantum fluid dynamics in threedimensional space. In continuation of the work reported previously, the GNLSE is applied to provide additional knowledge on the femtosecond dynamics of the electron density in the hydrogen molecule interacting with high-intensity laser fields. For this purpose, the GNLSE is solved numerically for many time-steps over a total interaction time of $100 \mathrm{fs}$, by employing a finite-difference scheme. Various time-dependent (TD) quantities, namely, electron density, ground-state survival probability and dipole moment have been obtained for two laser wavelengths and four different intensities. The high-order harmonics generation (HHG) is also examined. The present approach goes beyond the linear response formalism and, in principle, calculates the TD electron density to all orders of change.
\end{abstract}

Keywords. Hydrogen molecule; intense laser fields; femtosecond dynamics; time-dependent density; density functional theory; quantum fluid dynamics.

\section{Introduction}

In the last two decades, the multiphoton, femtosecond dynamics of strongly non-linear interaction of atoms with intense laser fields has received a great deal of attention. ${ }^{1-10} \mathrm{~A}$ wealth of new and sometimes counterintuitive phenomena has been discovered, involving the absorption of hundreds or thousands of photons by a single atom. Such phenomena include abovethreshold ionization (ATI), high-order harmonics generation (HHG), stabilization under superintense fields, etc. Considerable insights into these phenomena have been obtained through semi-classical and time-dependent (TD) quantum mechanical methods of varying degrees of sophistication. ${ }^{11-22}$ These included TD Hartree-Fock ${ }^{11}$ and TD density functional calculations. ${ }^{15-17}$ In view of the requirement of non-perturbative quantum mechanical methodologies for explaining the above phenomena, the most accurate method is the numerical solution of the TD Schrödinger equation for the problem. ${ }^{18-22}$ It

\footnotetext{
*Dedicated to Prof. D Mukherjee on his 60th birthday

**For correspondence
}

has also been noted that one- and two-dimensional non-linear oscillators can reproduce most features of the laser-atom interaction, ${ }^{19}$ including the possible occurrence of quantum chaos ${ }^{21,22}$ as a result of the interaction. Over the years, such experimental and theoretical studies have also resulted in a wide range of applications, e.g. the development of attosecond and X-ray lasers. 19,23

Very recently, strong interest has developed in the study of molecules under intense laser fields. ${ }^{24,25}$ Compared with atoms, the additional degrees of freedom associated with nuclear motion in molecules result in other interesting phenomena. Apart from ATI and HHG, these include above-threshold dissociation (ATD) ${ }^{24,25}$ alignment of the molecule with the laser field, ${ }^{26}$ ionization dependent on internuclear distance, ${ }^{27}$ bond dissociation dynamics, in particular, bond softening $^{28}$ and Coulomb explosion. ${ }^{29}$ A striking outcome of such studies has been the first experimental picture of a wavefunction, namely, the $3 \sigma_{g} \mathrm{HOMO}$ (highest occupied molecular orbital) of $\mathrm{N}_{2}$ molecule, obtained from the HHG spectra by using a tomographic algorithm. ${ }^{30}$

In a previous work from our laboratory, ${ }^{31}$ the dynamical electron density changes and HHG spectra of 
$\mathrm{H}_{2}$ molecule generated by intense laser fields were studied. The overall femtosecond dynamics were explained through the numerical solution of a TD generalized non-linear Schrödinger equation (GNLSE) derived earlier ${ }^{32}$ by combining density functional theory and quantum fluid dynamics in real space. Even though nuclear motion was not taken into account, interesting insights were obtained into the phenomena of excitation, ionization, bond softening, dipole formation and HHG. ${ }^{31}$ The method goes beyond the linear response formalism and, in principle, calculates the TD electron density to all orders of change.

The present paper is a continuation of the above work and therefore uses the same methodology. Here we report additional information on the changes in electron density, ground-state survival probability, TD dipole moment, etc. under intense laser fields of wavelengths 532 and $1064 \mathrm{~nm}$, as well as four different intensities, $1 \times 10^{14}, 2 \times 10^{14}, 3 \times 10^{14}$ and $1 \times$ $10^{15} \mathrm{~W} \mathrm{~cm}^{-2}$. The fast Fourier transform of the dipole moment yields the HHG spectrum.

Section 2 of this paper gives a brief account of the methodology employed (see ref. 31 for details). Section 3 presents and discusses the results while $\S 4$ makes a few concluding remarks.

\section{Methodology}

The $\mathrm{GNLSE}^{32}$ for a many-electron system is given by (atomic units employed throughout this paper)

$$
\left[-(1 / 2) \Delta+V_{\text {eff }}([\rho] ; \mathbf{r}, t)\right] \psi(\mathbf{r}, t)=i \partial \psi(\mathbf{r}, t) / \partial t,
$$

where $\psi(\mathbf{r}, t)$ is the hydrodynamical 'wavefunction' and the electron density $\rho(\mathbf{r}, t)=|\psi(\mathbf{r}, t)|^{2}$. The effective potential $V_{\text {eff }}([\rho] ; \mathbf{r}, t)$ consists of both classical and quantum terms; it is given by a sum of potential terms representing interelectronic Coulomb repulsion, electron-nuclear Coulomb attraction, exchange and correlation interactions, interaction with an external (e.g. laser) field and a non-classical kinetic energy correction term which vanishes for oneelectron systems and two-electron Hartree-Fock systems (in the case of $\mathrm{H}_{2}$ molecule, this correction term is neglected) but contributes significantly for systems containing more than two electrons. Equation (1) reduces to the Schrödinger equation for one-electron systems and to the TD Kohn-Sham equations for two-electron systems. It also bears a superficial resemblance to the Gross-Pitaevskii equation, a cubic
Schrödinger equation, ${ }^{33,34}$ for Bose-Einstein condensate, but is actually more complicated due to the presence of both non-integer powers of $\psi(\mathbf{r}, t)$ and an integral operator.

The detailed expressions for various potential terms in $V_{\text {eff }}$ have been given in. ${ }^{31}$ For the exchange potential, the Ghosh-Deb local functional ${ }^{35}$ has been employed while, for the correlation potential, a local Wigner-type functional ${ }^{31}$ has been used. Both these functionals give excellent exchange and correlation energies respectively for atoms and molecules.

The symmetry of the problem necessitates the use of a cylindrical coordinate system, $\tilde{\rho}, \tilde{z}, \varphi(0 \leq \tilde{\rho} \leq \infty$; $-\infty \leq \tilde{z} \leq+\infty ; 0 \leq \varphi \leq 2 \pi)$. The origin is at the midpoint of $R_{\mathrm{eq}}=1.4$ a.u. For numerical calculations, a finite computation grid was chosen, viz. $-25 \cdot 1250 \leq$ $\tilde{z} \leq+25 \cdot 1250,0 \leq x \leq 5.025$ a.u., where $x^{2}=\tilde{\rho}$, due to azimuthal symmetry, $\varphi$ is involved only when integration over the whole three-dimensional space is required. The mesh sizes in space and time are $\Delta x=\Delta \tilde{z}=0.1005$ a.u., $\Delta t=0.071643$ and 0.03582 a.u. for lasers of wavelengths 1064 and $532 \mathrm{~nm}$ respectively.

Thus, one optical cycle is completed in 2048 time steps. For solving (1), a finite-difference scheme was applied followed by Peaceman-Rachford splitting and a modified Thomas algorithm. A mask function was employed to take care of reflections from the grid boundaries. The computational details are given ref. 31. A more sophisticated algorithm for solving (1) in both imaginary time and real time has been developed very recently. ${ }^{36}$ Note that the present method goes beyond the linear response formalism and in principle calculates the TD electron density to all orders of change.

The laser electric field is applied along the $\tilde{Z}$-axis and is given by

$$
E(t)=E_{0} f(t) \sin \left(\omega_{\mathrm{L}} t\right),
$$

where the peak field $E_{0}=(8 \pi \mathrm{I} / \mathrm{c})^{1 / 2}, c$ is the speed of light, $I$ is the intensity of the laser field, $\omega_{\mathrm{L}}$ is the laser frequency and $f(t)$ is a dimensionless linear ramp function which reaches its peak value of unity at the end of five optical cycles. The computations cover 28 and 56 optical cycles for the 1064 and $542 \mathrm{~nm}$ lasers respectively, with $\omega_{\mathrm{L}}=0.042823$ and 0.085646 a.u. for these two lasers. Thus, the total laser-molecule interaction time considered is $4134 \cdot 1276$ a.u. (1 a.u. of time $=0.0241889 \mathrm{fs}=24.1889$ as), i.e. $100 \mathrm{fs}$. 
For launching the computations at $t=0$, the input is the ground-state density of $\mathrm{H}_{2}$ molecule. This is computed from (1) through evolution in imaginary time, similar to the diffusion quantum Monte Carlo method. This technique yields a quite accurate, beyond Hartree-Fock, molecular electron density with the ground-state energy of -1.17212 a.u., compared to the exact non-relativistic energy of $-1 \cdot 17447$ a.u. ${ }^{31}$
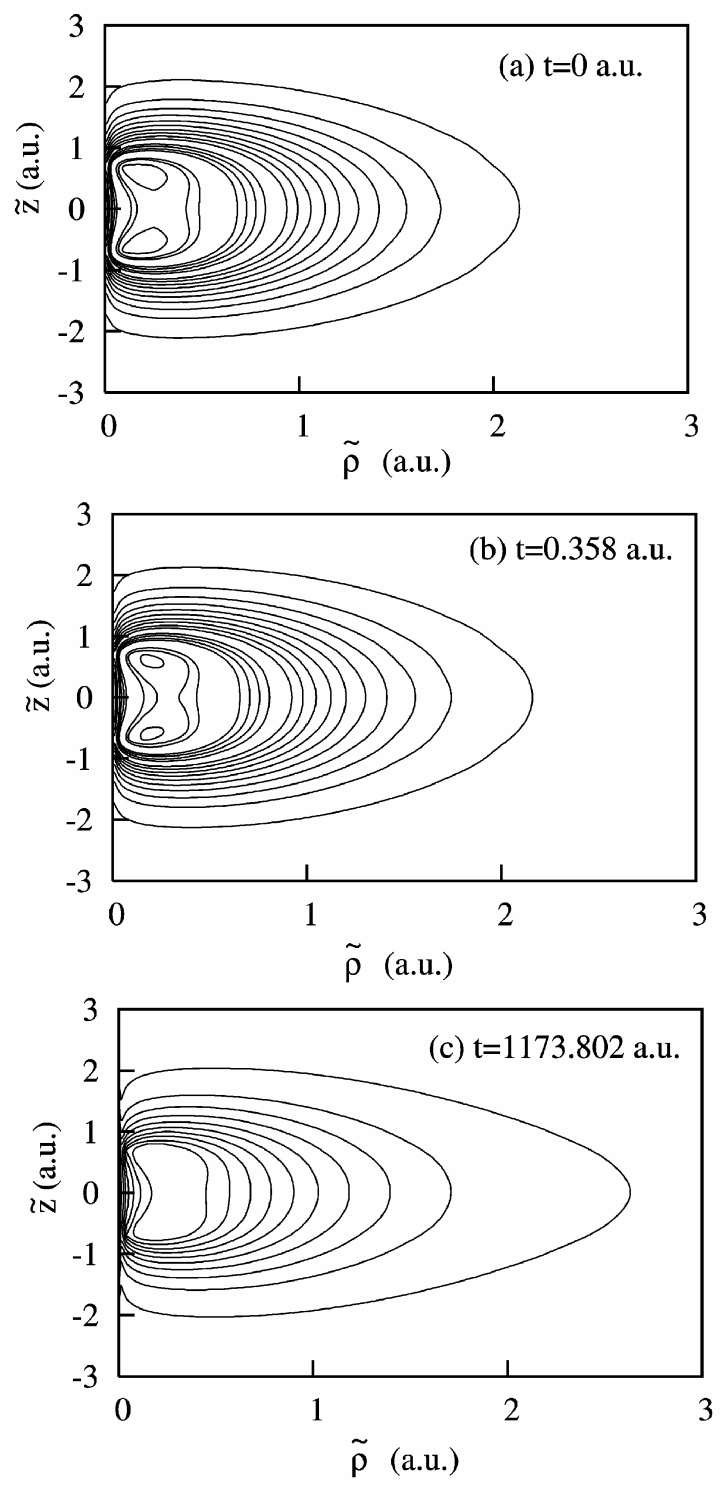

Figure 1. Contours of the time-dependent electron density (a.u.) in the $\tilde{\rho} \tilde{z}$-plane for $\lambda_{\mathrm{L}}=532 \mathrm{~nm}, I=1 \times$ $10^{14} \mathrm{~W} \mathrm{~cm}^{-2}$, at (a) $t=0$ (reproduced by permission from ref. 31 ), (b) $t=0.358$ a.u. (8.66 as) and (c) $t=1173.802$ a.u. (16 optical cycles, zero field; one optical cycle covers $t=73 \cdot 3626$ a.u.). In figures $1-3$, the density values for the innermost and outermost contours are 0.22 and 0.008 a.u.

\section{Results and discussion}

Below, we present results for different laser wavelengths and intensities for the TD electron density, ground-state survival probability and dipole moment as well as HHG spectra.

Figure 1 depicts the TD electron density for $\lambda_{\mathrm{L}}=$ $532 \mathrm{~nm}$ and $I=1 \times 10^{14} \mathrm{Wcm}^{-2}$ at three different times, including the initial, $t=0$ (figure 1a). The initial density is symmetric about the origin. Since the laser field is aligned along the $\tilde{z}$-axis, beginning from the positive side, a pronounced up-down asymmetry in the density occurs along the $\tilde{z}$-axis, even during the ramp, creating a TD dipole moment. When the laser field proceeds towards its peak value $\left(+E_{0}\right)$, the density asymmetry develops upwards along the positive $\tilde{z}$-axis. When the field proceeds towards its minimum value $\left(-E_{0}\right)$, the density asymmetry develops in the downward direction along the negative $\tilde{z}$-axis. Both these effects are caused by the attractive potential from the up-and-down swinging laser field. Since the present formalism is capable of detecting attosecond changes in the electron density, the density contours, especially the inner ones, at $t=0.358$ a.u. ( $\sim 9$ as) are slightly different from those at $t=0$ (figure 1b). At $t=1173.802$ a.u. (figure $1 \mathrm{c}$ ), sixteen optical cycles have elapsed and, since the laser field is now zero, the up-down symmetry of the density is again restored. This also serves as a numerical check on our computations. Figure 1c also shows ionization from both a reduction in the inner contours and a spread in density. Although nuclear motion is not taken into account in this work, Figure 1c shows clear evidence of bond softening since density in the binding region ${ }^{37}$ is reduced. Continuing and stronger

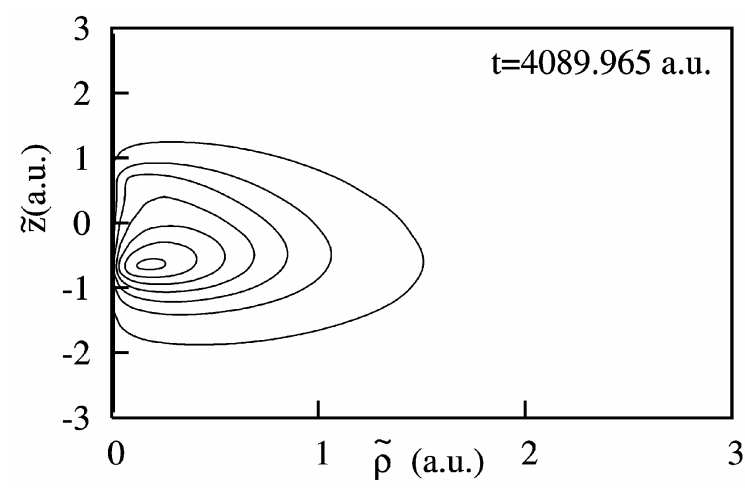

Figure 2. Contours of the time-dependent electron density (a.u.) in the $\tilde{\rho} \tilde{z}$-plane for $\lambda_{\mathrm{L}}=532 \mathrm{~nm}, I=1 \times$ $10^{15} \mathrm{~W} \mathrm{~cm}^{-2}, t=4089.965$ a.u. (the trough of the last optical cycle). Compare with figure 1 . 
evidence of both ionization and bond softening can be seen in figure 2 which depicts fewer density contours at $t=4089.965$ a.u. (the trough of the last optical cycle), $\lambda_{\mathrm{L}}=532 \mathrm{~nm}$ and $I=1 \times 10^{15} \mathrm{~W} \mathrm{~cm}^{-2}$. The depletion of electron density around both protons is a precursor to Coulomb explosion.

Figure 3 visually compares the increase in the rate of ionization of the molecule with an increase in the laser intensity, for $\lambda_{\mathrm{L}}=1064 \mathrm{~nm}$, at $t=3998.262$ a.u., i.e. after the 27 th optical cycle. At $I=1 \times$
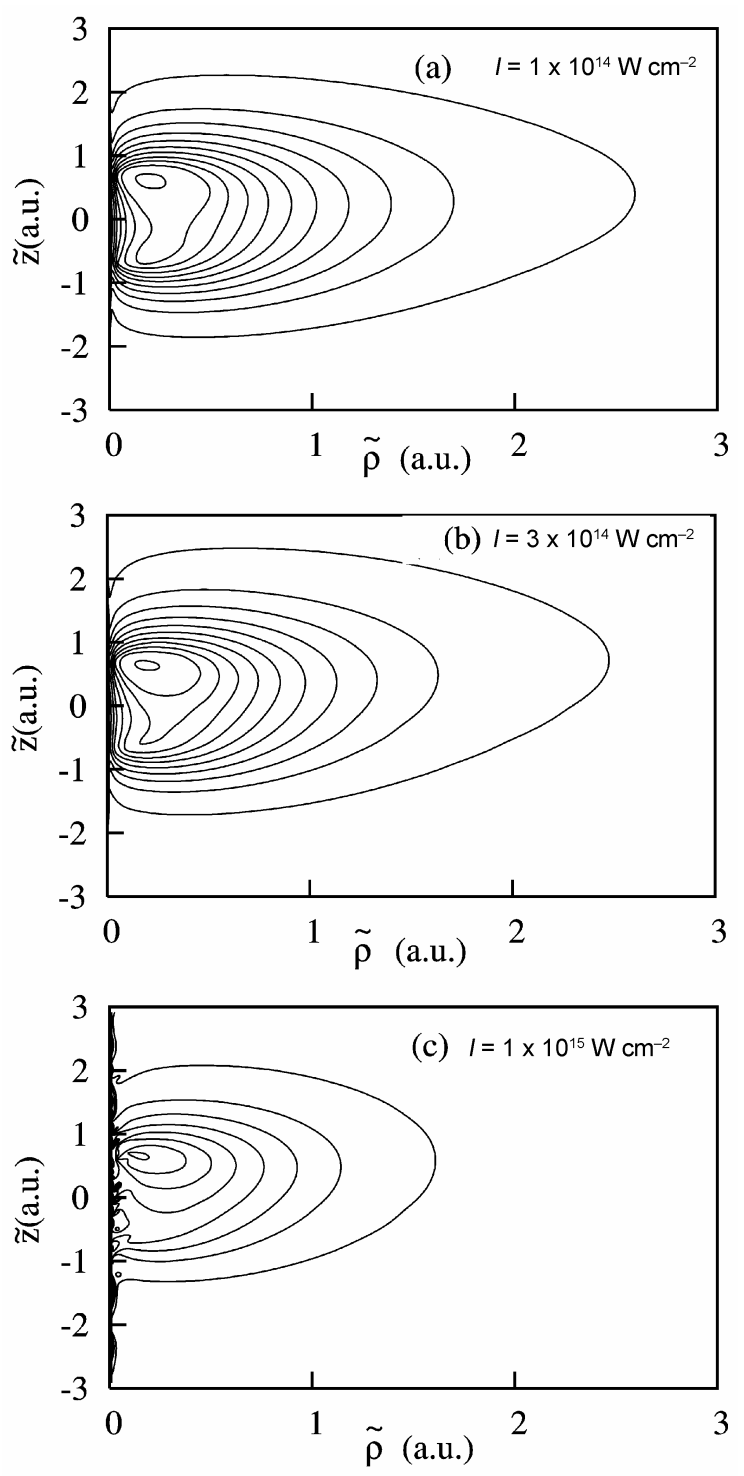

Figure 3. Contours of the time-dependent electron density (a.u.) in the $\tilde{\rho} \tilde{z}$-plane for $\lambda_{\mathrm{L}}=1064 \mathrm{~nm}, t=3998 \cdot 262$ a.u. (27.25 optical cycles; one optical cycle covers $t=$ $146 \cdot 7252$ a.u.), for (a) $I=1 \times 10^{14} \mathrm{Wcm}^{-2}$, (b) $I=3 \times$ $10^{14} \mathrm{Wcm}^{-2}$, and (c) $I=1 \times 10^{15} \mathrm{Wcm}^{-2}$. See figure 1 .
$10^{14} \mathrm{Wcm}^{-2}$ (figure $3 \mathrm{a}$ ), the number of density contours has decreased compared to the density at $t=0$ (figure 1a), indicating ionization. In contrast, the rate of ionization up to this time is greater for $I=3 \times 10^{14} \mathrm{Wcm}^{-2}$ (figure $3 \mathrm{~b}$ ). Since the positive charge on the molecule's ionic core is now greater compared to figure $3 \mathrm{a}$, the density contracts towards the nuclei. This situation is further enhanced at $I=1 \times 10^{15} \mathrm{Wcm}^{-2}$ (figure 3c) where the rate of ionization up to this time is still greater, as indicated by the smaller number of density contours and further contraction of the density towards the nuclei. The density asymmetry along the positive $\tilde{z}$-axis occurs since the laser electric field is now positive and the corresponding potential is attractive (see above). Thus, figures 1-3 together communicate a picture of ionization, bond softening and Coulomb explosion.

Figure 4 depicts the ground-state survival probability, $P_{\mathrm{gs}}(t)$ for two laser wavelengths and four different laser intensities. $P_{\mathrm{gs}}(t)$ is given by

$$
P_{\mathrm{gs}}(t)=|\langle\psi(\mathbf{r}, t) \mid \psi(\mathbf{r}, 0)\rangle|^{2},
$$

and is normalized to unity at $t=0$. It is obvious that due to excitation and ionization, $P_{\mathrm{gs}}(t)$ would rapidly decrease from unity. Figure 4 shows the oscillatory changes in $P_{\mathrm{gs}}(t)$, in phase with oscillations in the laser field, the pattern of oscillations depending on both laser frequency and intensity. Further decreases in $P_{g s}(t)$ result from ionization occurring after the ramp is reached. Note that the oscillation pattern for $\lambda_{\mathrm{L}}=532 \mathrm{~nm}$ and $I=2 \times 10^{14} \mathrm{Wcm}^{-2}$ (figure $4 \mathrm{a}$ ) is similar to that for $\lambda_{\mathrm{L}}=1064 \mathrm{~nm}$ but at a higher intensity, viz. $3 \times 10^{14} \mathrm{Wcm}^{-2}$ (figure $4 \mathrm{~d}$ ). Figures 4(b-e) show that for $\lambda_{\mathrm{L}}=1064 \mathrm{~nm}$, the magnitude of the decrease in $P_{\mathrm{gs}}(t)$ increases with an increase in laser intensity. This implies that excitation to higher states occurs faster with increased laser intensity. Furthermore, a comparison of the rates of ionization with those of the changes in $P_{\mathrm{gs}}(t)$ shows that excitation precedes the onset of ionization. However, at later times, both excitation and ionization proceed together.

At this stage, one recalls that the laser field is linearly polarized along the internuclear axis, i.e. the $\tilde{z}$-direction. The TD induced dipole moment is therefore given by

$$
\mu_{\tilde{z}}(t)=\int \rho(\tilde{\rho}, \tilde{z} t) \tilde{z} \tilde{\rho} \mathrm{d} \tilde{\rho} \mathrm{d} \tilde{z} \mathrm{~d} \varphi .
$$

Figures $5(\mathrm{a}, \mathrm{b})$ show that $\mu_{\tilde{z}}(t)$ also oscillates in phase with the laser field and, as time progresses, 

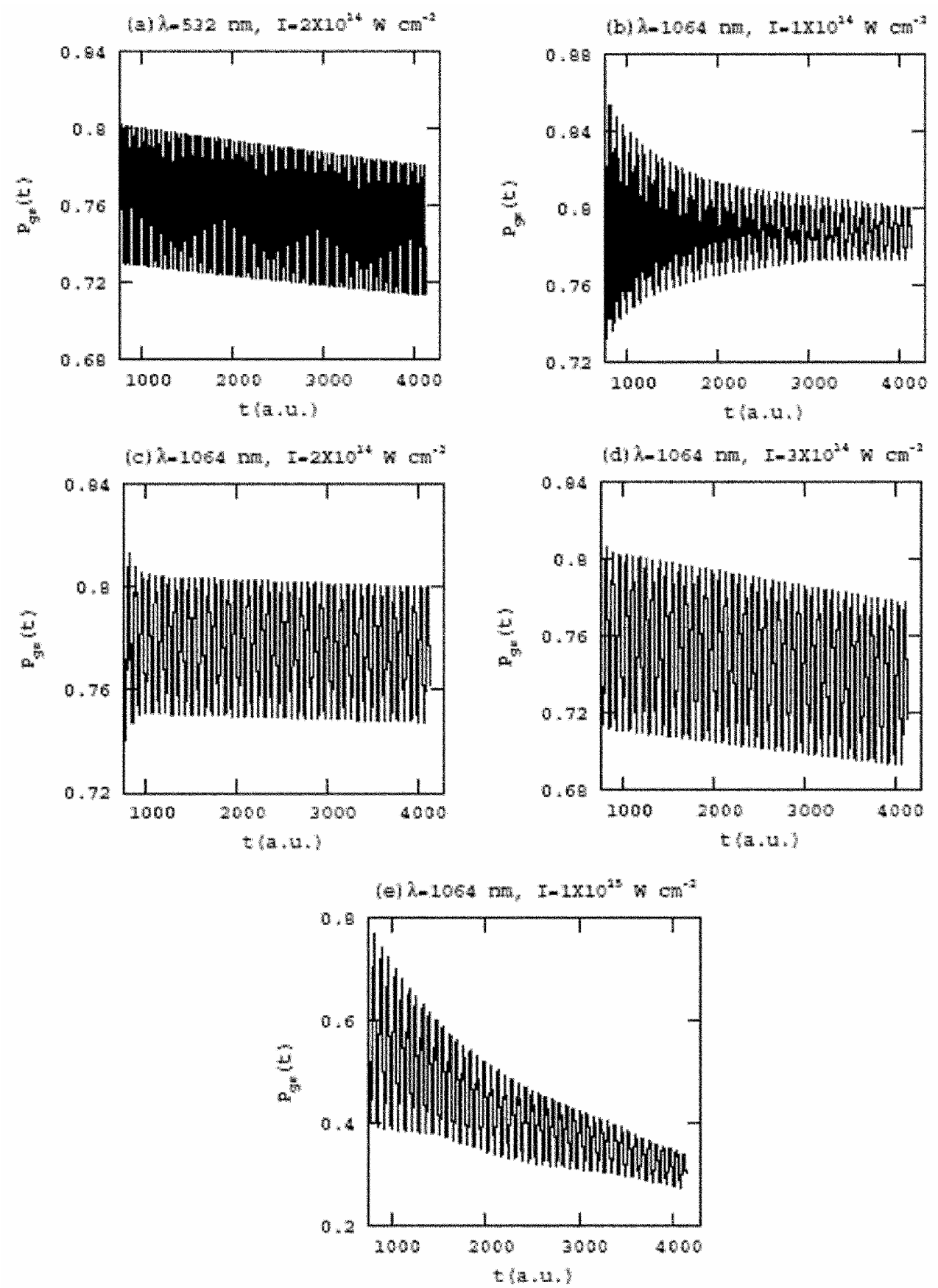

Figure 4. Ground-state survival probability, $P_{\mathrm{gs}}(t)$ for (a) $\lambda_{\mathrm{L}}=532 \mathrm{~nm}, \quad I=2 \times$ $10^{14} \mathrm{Wcm}^{-2}$; (b) $\lambda_{\mathrm{L}}=1064 \mathrm{~nm}, I=1 \times 10^{14} \mathrm{Wcm}^{-2}$; (c) $\lambda_{\mathrm{L}}=1064 \mathrm{~nm}, I=2 \times 10^{14} \mathrm{Wcm}^{-2}$; (d) $\lambda_{\mathrm{L}}=1064 \mathrm{~nm}, I=3 \times 10^{14} \mathrm{Wcm}^{-2}$; and (e) $\lambda_{\mathrm{L}}=1064 \mathrm{~nm}, I=1 \times 10^{15} \mathrm{Wcm}^{-2}$.

the amplitude of oscillation decreases since electron density is progressively lost due to ionization. This damping is more pronounced for $I=1 \times 10^{15} \mathrm{Wcm}^{-2}$ (figure $5 \mathrm{c}$ ). Note that an increase in polarity of the molecule facilitates excitation and ionization and therefore both bond softening and Coulomb explosion.

Finally, figure 6 depicts the HHG spectra for the $532 \mathrm{~nm}$ and $1064 \mathrm{~nm}$ lasers ${ }^{31}$ at $I=1 \times 10^{15} \mathrm{Wcm}^{-2}$. This shows the usual pattern of HHG, viz. a rapid 

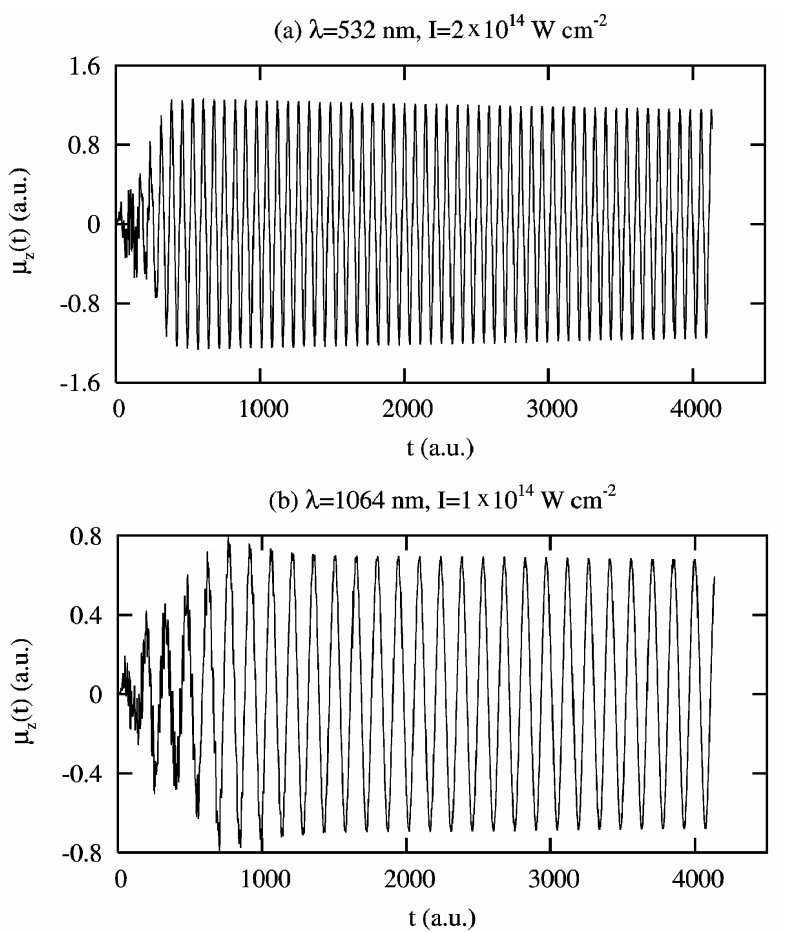

(c) $\lambda=1064 \mathrm{~nm}, \mathrm{I}=1 \times 10^{15} \mathrm{~W} \mathrm{~cm}^{-2}$

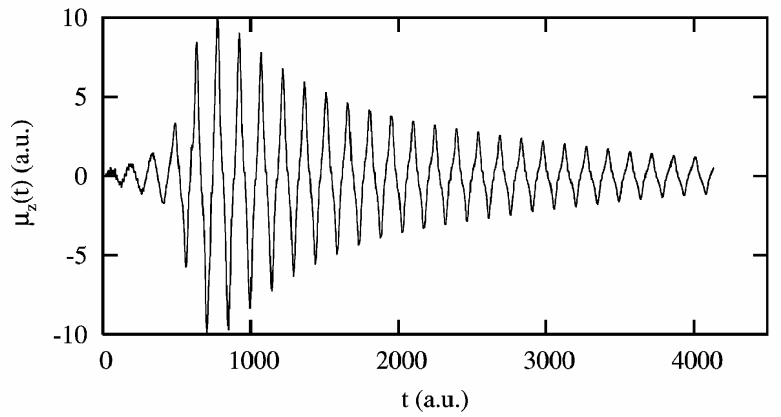

Figure 5. Time-dependent dipole moment (a.u.), $\mu_{\tilde{z}}(t)$ for (a) $\lambda_{\mathrm{L}}=532 \mathrm{~nm}, I=2 \times 10^{14} \mathrm{Wcm}^{-2}$; (b) $\lambda_{\mathrm{L}}=1064 \mathrm{~nm}$, $I=1 \times 10^{14} \mathrm{Wcm}^{-2}$; (c) $\lambda_{\mathrm{L}}=1064 \mathrm{~nm}, I=1 \times 10^{15} \mathrm{Wcm}^{-2}$.

fall for the first few harmonics followed by a plateau which terminates in a sharp cut-off. As expected, only odd harmonics appear due to the initial centrosymmetry of the molecule; it has, however, been observed and explained that this symmetry selection rule can be violated at higher laser frequencies and/or intensities where even harmonics can also appear near the end of the plateau. ${ }^{19}$ The number of harmonics for $\lambda_{\mathrm{L}}=1064 \mathrm{~nm}$ is nearly double the number of harmonics for $\lambda_{\mathrm{L}}=532 \mathrm{~nm}$, indicating that the energy output remains about the same at the same laser intensity. Such high harmonics provide an excellent and efficient way of producing soft X-ray and XUV lasers as well as attosecond pulses. ${ }^{19,23}$
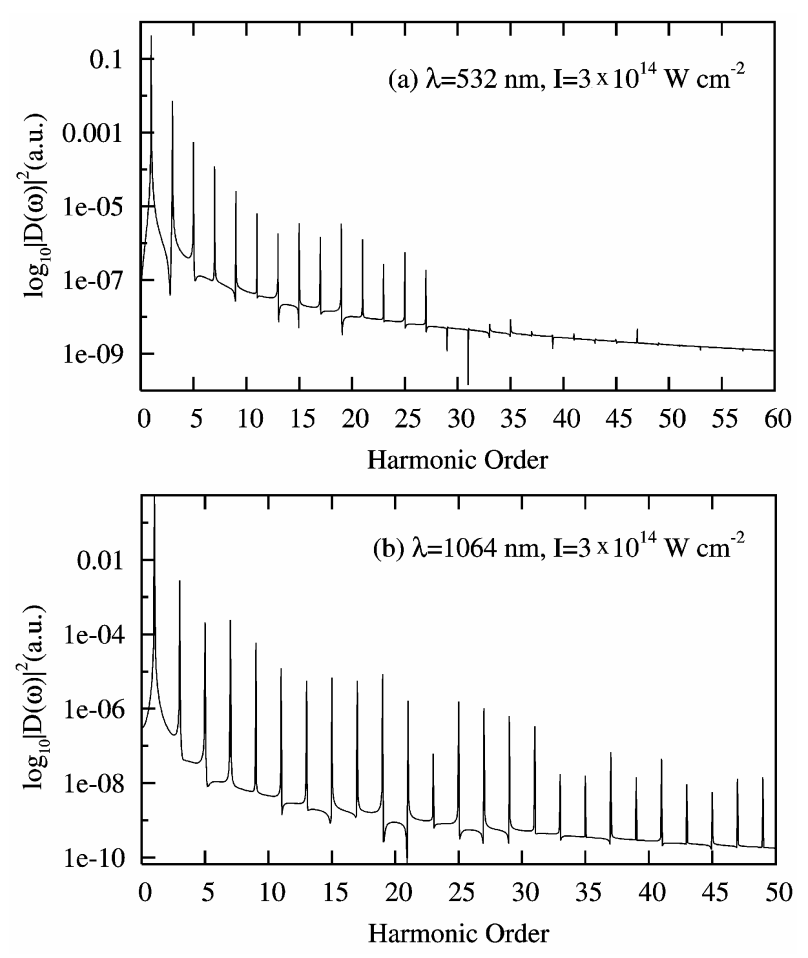

Figure 6. A comparison of the $\mathrm{HHG}$ spectra at $I=3 \times 10^{14} \mathrm{Wcm}^{-2}$ for (a) $\lambda_{\mathrm{L}}=532 \mathrm{~nm}$, and (b) $\lambda_{\mathrm{L}}=$ $1064 \mathrm{~nm}$ (reproduced by permission (ref. 31)).

\section{Conclusion}

By numerically solving the GNLSE, (1), for the interaction between an $\mathrm{H}_{2}$ molecule at $R_{\text {eq }}$ and intense laser fields of different frequencies and intensities, additional information (see ref. 31) is provided here on several consequences of this interaction. Since only one equation is solved, including both exchange and correlation functionals, irrespective of how many electrons are present in the system, the present theoretical approach appears to have considerable potential in dealing with larger atoms/molecules interacting with intense laser fields.

\section{Acknowledgement}

Much of this work was done while one of us (A W) was at the Department of Chemistry, Panjab University, Chandigarh. We thank Prof. Ramesh Kapoor, Chairman of the Department, for the computation facilities. Financial support to A W from Council of Scientific and Industrial Research, New Delhi is gratefully acknowledged. 


\section{References}

1. Gavrila M (ed.) 1992 Atoms in intense laser fields (New York: Academic Press)

2. Mittleman M H 1993 Introduction to the theory of laseratom interactions (New York: Plenum Press)

3. Faisal F H M 1987 Theory of multiphoton processes (New York: Plenum Press)

4. Burnett K, Reed V C and Knight P L 1993 J. Phys. B: At. Mol. Opt. Phys. 26561

5. Protopapas M, Keitel C H and Knight P L 1997 Rep. Prog. Phys. 60389

6. Joachain C J, Dörr M and Kylstra N J 2000 Adv. At. Mol. Opt. Phys. 42225

7. Eberly J H, Grobe R, Law C K and Su Q 1992 Adv. At. Mol. Opt. Phys. Suppl. 1301

8. Eberly J H and Kulander K C 1993 Science 2621229

9. Piraux B and Rzazewski K (eds) 2001 Super-intense laser-atom physics (Dordrecht: Kluwer Academic)

10. Batani D, Joachain C J, Martelluci S and Chester A N (eds) 2001 Atoms, solids and plasmas in superintense laser fields (Dordrecht: Kluwer Academic)

11. Kulander K C 1987 Phys. Rev. A36 2726

12. Rae S C, Chen X and Burnett K 1994 Phys. Rev. A50 1946

13. Bandarage G, Maquet A, Ménis T, Taieb R, Véniard V and Cooper J 1992 Phys. Rev. A46 380

14. Kaplan A E and Shkolnikov P L 1994 Phys. Rev. A49 1275

15. Petersilka M and Gross E K U 1999 Laser Phys. 9 105

16. Dey B K and Deb B M 1995 Int. J. Quant. Chem. 56 707

17. Dey B K and Deb B M 1998 Int. J. Quant. Chem. 70 441

18. Taylor K T, Parker J S, Dundas D, Smyth E and Vivirito S 1999 in Non-linear dynamics and compu- tational physics (ed.) V B Sheorey (New Delhi: Narosa Publishing)

19. Wadehra A, Vikas and Deb B M 2003 J. Chem. Phys. 1196620

20. Wadehra A and Deb B M 2003 Proc. Indian Acad. Sci. (Chem. Sci.) 115 349; Wadehra A and Deb B M 2004; Erratum J. Chem. Sci. 116129

21. Gupta N and Deb B M 2006 Chem. Phys. 327351

22. Gupta N and Deb B M 2006 Pramana-J. Phys. 67 1055

23. Mairesse Y, de Bohan A, Frasinski L J, Merdji H, Dinu L C, Monchicourt P, Preger P, Kovacev M, Taieb R, Carré B, Muller H G, Agostini $\mathrm{P}$ and Saliéres P 2003 Science 3021540

24. Bandrauk A D (ed.) 1994 Molecules in laser fields (New York: Marcel Dekker)

25. Posthumus J H 2004 Rep. Prog. Phys. 67623

26. Larsen J J, Wendt-Larsen I and Stapelfeldt H 1999 Phys. Rev. Lett. 831123

27. Saenz A 2000 Phys. Rev. A61 051402(R)

28. Saenz A 2002 Phys. Rev. $A 66063407$

29. Constant E, Stapelfeldt H and Corkum P B 1996 Phys. Rev. Lett. 764140

30. Itatani J, Levesque J, Zeidler D, Niikura H, Pépin H, Kieffer J C, Corkum P B and Villeneuve D M 2004 Nature 432867

31. Wadehra A and Deb B M 2006 Eur. Phys. J. D39 141

32. Deb B M and Chattaraj P K 1989 Phys. Rev. A39 1696

33. Gross E P 1961 Nuovo Cim. 20454

34. Pitaevskii L P 1961 Sov. Phys. - JETP 13451

35. Ghosh S K and Deb B M 1994 J. Phys. B: At. Mol. Opt. Phys. 27381

36. Poddar A and Deb B M 2007 J. Phys. A: Math. Theor. 405981

37. Bader R F W 1981 in The force concept in chemistry (ed.) B M Deb (New York: Van Nostrand-Reinhold) 